Revista de Matemática: TeORÍA y AplicaCiones 2015 22(1) : 61-69

CIMPA - UCR ISSN: 1409-2433 (PRINT), 2215-3373 (ONLINE)

\title{
WAVELETS INFINITAMENTE OSCILANTES Y UN EFICIENTE ALGORITMO DE IMPLEMENTACIÓN BASADO EN LA FFT
}

\section{INFINITELY OSCILLATING WAVELETS AND AN \\ EFFICIENT IMPLEMENTATION ALGORITHM BASED ON THE FFT}

\author{
Marcela FAbio* $^{*}$ Eduardo P. SERranO $^{\dagger}$
}

Received: 5/Mar/2012; Revised: 3/Sep/2014;

Accepted: $17 /$ Oct/2014

${ }^{*}$ Centro de Matemática Aplicada, Universidad de San Martín, Argentina. E-Mail: mfabio@unsam.edu.ar

${ }^{\dagger}$ Centro de Matemática Aplicada, Universidad de San Martín y Escuela Superior Técnica del Ejército "General Manuel N. Savio”, I.E.S.E., Argentina. E-Mail: eserrano@unsam.edu.ar 


\title{
Resumen
}

En este trabajo presentamos el diseño de una wavelet ortogonal, infinitamente oscilante, localizada en el tiempo con decaimiento $1 /|t|^{n}$ y de banda limitada. Su aplicación conduce a la descomposición de señales en ondas de frecuencia instantánea bien definida. Presentamos además el algoritmo de implementación para el análisis y síntesis basado en la Transformada Rápida de Fourier con la misma complejidad que el algoritmo de Mallat.

Palabras clave: wavelet tipo pasa-banda; algoritmo de Mallat; FFT; análisis de multirresolución; frecuencia instantánea.

\begin{abstract}
In this work we present the design of an orthogonal wavelet, infinitely oscillating, located in time with decay $1 /|t|^{n}$ and limited-band. Its application leads to the signal decomposition in waves of instantaneous, well defined frequency. We also present the implementation algorithm for the analysis and synthesis based on the Fast Fourier Transform (FFT) with the same complexity as Mallat's algorithm.
\end{abstract}

Keywords: pass-band wavelet; Mallat's algorithm; FFT; multiresolution analysis; instantaneous frequency.

Mathematics Subject Classification: 42C40, 44A05.

\section{Introducción}

La transformada wavelet (o en onditas) juega un relevante papel en las aplicaciones numéricas, principalmente en el campo del procesamiento de señales e imágenes, [5], [6].

Consideramos una señal $f \in L^{2}(\mathbb{R})$ en la que conviven patrones oscilantes, fenómenos transitorios y elementos sin estructura.

La transformada discreta basada en una wavelet ortonormal $\psi$, organiza la completa información de una señal $f$, en los respectivos coeficientes wavelets, localizados en tiempo y en escala.

Denotamos $\psi_{j k}(t)=2^{j / 2} \psi\left(2^{j} t-k\right)$ y $c_{j k}(f)=<f, \psi_{j k}>$.

La información contenida en tales coeficientes o átomos, puede procesarse posteriormente aplicando técnicas de filtrado, compresión o síntesis selectiva o mediante técnicas de reconocimiento de patrones para caracterizar fenómenos de compleja estructura.

Una cuestión siempre abierta en el campo de las aplicaciones numéricas es la elección de la wavelet madre $\psi$. Se pretende que la misma sea una función 
oscilante, bien localizada en tiempo y frecuencia y asociada con un eficiente cálculo numérico computacional.

Si la wavelet es ortogonal no es posible optimizar simultáneamente todas las propiedades requeridas y su elección dependerá del problema específico a resolver.

Usualmente se emplean las wavelets de Daubechies, ortogonales de soporte compacto, con un número finito de momentos nulos, pero de regularidad fraccionaria.

Otra opción son las wavelets splines de orden impar, afines, cúbicas o quínticas, muy eficientes desde el punto de vista computacional.

En ambos casos, el cálculo de los coeficientes se realiza mediante el algoritmo recursivo de Mallat, basado en la aplicación recursiva del par de filtros conjugados asociados a la relación de doble escala de la correspondiente función de escala.

Este algoritmo tiene la misma complejidad que el algoritmo FFT. Este esquema algorítmico puede extenderse a bancos de filtros para una mejor localización en frecuencia o pares de filtros de perfecta reconstrucción no ortogonales.

En algunas aplicaciones se requiere de wavelets infinitamente oscilantes, ortogonales, bien localizadas en tiempo y frecuencia. Un ejemplo esquemático de tales wavelets son la wavelet de Shannon o la wavelet de Meyer, estas son infinitamente oscilantes pero no están bien localizadas en el tiempo, siendo prácticamente inaplicables.

En la literatura [4] se propone calcular los coeficientes con la wavelet de Shannon en el dominio de las frecuencias utilizando la FFT (Transformada Rápida de Fourier o Fast Fourier Transform en inglés).

Esto no mejora la mala localización temporal de la transformada de Shannon, pero sugiere aplicar un esquema similar utilizando una especial tipo de wavelet denominada pasa-banda, [10].

Con esta finalidad presentamos en este trabajo el diseño de una wavelet ortogonal con las siguientes propiedades:

1. $\widehat{\psi}$ soportada en la banda bilateral $(\pi-\pi / m)<|\omega|<2(\pi+\pi / m)$, con $m \geq 3$,

2. $\widehat{\psi}$ es $\mathbb{C}^{n+1}$, con $n \geq 7$,

3. $\left\{\psi_{j k}, j, k \in \mathbb{Z}\right\}$ constituye una base ortonormal de $L^{2}(\mathbb{R})$.

Esta wavelet es infinitamente oscilante, localizada en el tiempo y decae como $\frac{1}{|t|^{n}}$. Puede ser empleada para el estudio de regularidad de señales, estimación de frecuencias instantáneas combinadas con la transformada Hilbert 
[10], o para descomponer en ondas del tipo funciones modales intrínsecas, cuasimonocromáticas de frecuencia bien definida, [2].

Presentamos también el algoritmo para el análisis y la síntesis, basado en la Transformada rápida de Fourier (FFT).

\section{Wavelets de Malvar-Wilson-Meyer}

El diseño propuesto se realiza en el contexto de un Análisis de Multirresolución gene- rado por wavelets del tipo de Malvar-Wilson-Meyer, [6]. Para las señales dadas por un conjunto finito de datos, las operaciones de análisis y síntesis se realizan en el dominio de las frecuencias mediante la aplicación de eficientes algoritmos basados en la FFT.

Dada la partición de la recta real en intervalos

$$
\left[a_{j}, a_{j+1}\right], \text { con } \cdots<a_{-1}<a_{0}<a_{1}<\cdots,
$$

donde $j \in \mathbb{Z}, l_{j}=a_{j+1}-a_{j}$ y $\alpha_{j}>0$ suficientemente pequeños, se diseñan las ventanas $w_{j}(t)=w\left(2^{-j} t\right)$, que verifican las siguientes condiciones:

- $w_{j}(t)=1$ si $a_{j}+\alpha_{j} \leq t \leq a_{j+1}-\alpha_{j+1}$.

- $w_{j}(t)=0$ si $t \leq a_{j}-\alpha_{j}$ ó $t \geq a_{j+1}+\alpha_{j+1}$.

- $w_{j}^{2}\left(a_{j}+\nu\right)+w_{j}^{2}\left(a_{j}-\nu\right)=1$ si $|\nu| \leq \alpha_{j}$.

- $w_{j-1}\left(a_{j}+\nu\right)=w_{j}\left(a_{j}-\nu\right)$ si $|\nu| \leq \alpha_{j}$.

La familia de funciones

$$
u_{j k}(t)=\sqrt{\frac{2}{l_{j}}} w_{j}(t) \cos \left(\frac{\pi}{l_{j}}(k+1 / 2)\left(t-a_{j}\right)\right), j \in \mathbb{Z}, k \in \mathbb{N}_{0},
$$

constituye base ortonormal de $L^{2}\left(\mathbb{R}_{\geq 0}\right)$. Una base análoga obtenemos utilizando senos.

Tomando $\omega>0$ como variable y $a_{j}=2^{j} \pi, \alpha_{j}=2^{j} r, 0<r \leq \pi / 3$, las siguientes funciones también son base ortonormal de $L^{2}\left(\mathbb{R}_{\geq 0}\right)$ en el dominio frecuencial,

$$
\begin{aligned}
& u_{j k}(\omega)=\sqrt{\frac{2^{1-j}}{\pi}} w_{j}(\omega) \cos \left(2^{j}(k+1 / 2)\left(\omega-2^{j} \pi\right)\right), j \in \mathbb{Z}, k \in \mathbb{N}_{0}, \\
& v_{j k}(\omega)=\sqrt{\frac{2^{1-j}}{\pi}} w_{j}(\omega) \sin \left(2^{j}(k+1 / 2)\left(\omega-2^{j} \pi\right)\right), j \in \mathbb{Z}, k \in \mathbb{N}_{0} .
\end{aligned}
$$

Rev.Mate.Teor.Aplic. ISSN 1409-2433 (Print) 2215-3373 (Online) Vol. 22(1): 61-69, Jan 2015 
Extendiendo por paridad e imparidad, respectivamente, para $\omega<0$, la familia de funciones

$$
\left\{\frac{1}{2}\left(u_{j k}+v_{j k}\right), \frac{1}{2}\left(u_{j k}-v_{j k}\right), \quad j \in \mathbb{Z}, k \in \mathbb{N}_{0}\right\}
$$

es base ortonormal de $L^{2}(\mathbb{R})$. Concluimos que la familia

$$
\left\{\sqrt{\frac{1}{2 \pi}} w_{j}(\omega) e^{-i(k+1 / 2) \omega}, j, k \in \mathbb{Z}\right\}
$$

resulta también una base ortonormal de $L^{2}(\mathbb{R})$, [6].

\section{La transformada wavelet}

Tomando $\alpha=\pi / m, m \geq 3$, y la correspondiente ventana para $j=0, w_{\alpha}(\omega)$, resulta la wavelet madre

$$
\widehat{\psi}(\omega)=w_{\alpha}(\omega) e^{-i \omega / 2}=|\widehat{\psi}(\omega)| e^{-i \omega / 2}
$$

que es nula $|\omega| \geq[\pi-\alpha, 2 \pi+2 \alpha]$. Luego la familia

$$
\psi_{j k}(t)=2^{j / 2} \psi\left(2^{j} t-k\right), \quad j, k \in \mathbb{Z}
$$

es una base ortonormal de wavelets de $L^{2}(\mathbb{R})$. Entonces, si $f_{j} \in W_{j}$,

$$
\widehat{f}_{j}(\omega)=2^{-j / 2} w_{\alpha}\left(\omega / 2^{j}\right) \sum_{k} c_{j k} e^{-i \omega(k+1 / 2) / 2^{j}}
$$

siendo $c_{j k} \in l^{2}(\mathbb{R})$ los coeficientes wavelets.

La Figura 1 muestra el gráfico de la wavelet para $\alpha=\pi / 7$.

\section{Subwavelets}

A continuación proponemos el siguiente refinamiento utilizando apropiadas subventanas que realizan la partición:

$$
w_{\alpha}(\omega)=w_{\alpha}^{(1)}(\omega)+\cdots+w_{\alpha}^{(m)}(\omega)
$$

en una descomposición del tipo Littlewood-Paley, [7], [8].

Rev.Mate.Teor.Aplic. ISSN 1409-2433 (Print) 2215-3373 (Online) Vol. 22(1): 61-69, Jan 2015 


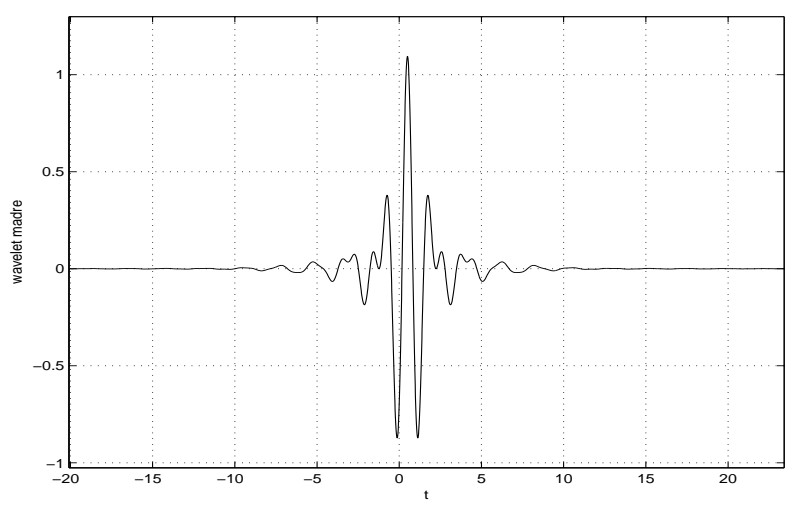

Figura 1: Wavelet madre.

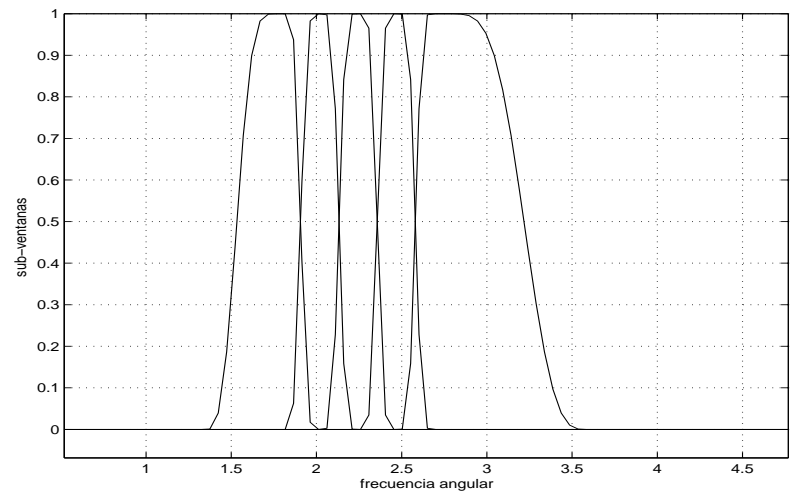

Figura 2: Subventanas, $j=-1, m=5$ y $1 \leq p \leq 5$.

Para $\alpha=\frac{\pi}{m+2}$ y $m \in \mathbb{N}_{>2}$, las subventanas $w_{\alpha}^{(p)}(\omega)$ están localizadas en torno a la frecuencia

$$
\omega=\pi\left(1+\frac{p}{m+2}\right), 1 \leq p \leq m .
$$

La Figura 2 muestra el gráfico de las subventanas en el nivel $j=-1, m=5$ y $1 \leq p \leq 5$.

En consecuencia, las subwavelets $\psi_{\alpha}^{(p)}(t)$ se definen como

$$
\widehat{\psi}_{\alpha}^{(p)}(\omega)=w_{\alpha}^{(p)}(\omega) e^{-i \omega / 2} .
$$

El gráfico de algunas subwavelets para el nivel $j=-1, m=5$ y $1 \leq p \leq 3$, se muestra en la Figura 3. 


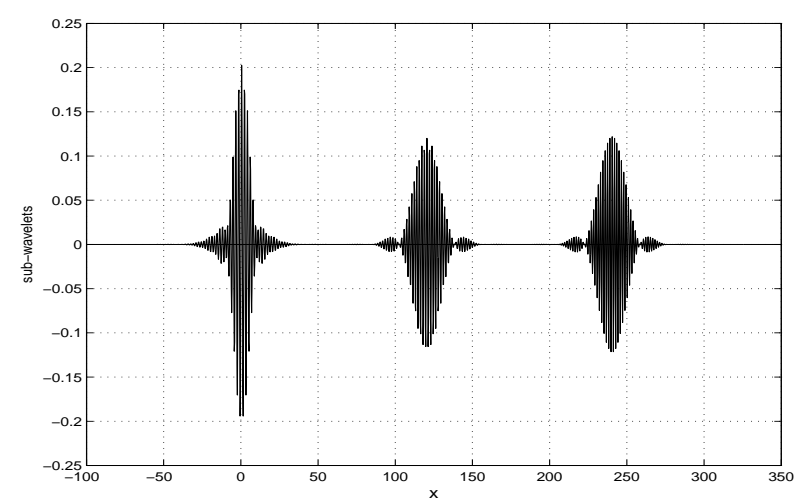

Figura 3: Subwavelets, $j=-1, m=5$ y $p=1,2,3$.

Entonces,

$$
\widehat{f}_{j}(\omega)=\sum_{p=1}^{m} \sum_{k \in Z} c_{j k} \widehat{\psi}_{j k}^{(p)}(\omega)=\sum_{p=1}^{m} f_{j}^{(p)}(\omega) .
$$

de esta forma las funciones $f_{j}^{(p)}(\omega)$ se asemejan a funciones modales intrínsecas y son casiortogonales.

La colección de subwavelets resulta sobrecompleta en cada $W_{j}$.

\section{$5 \quad$ Algoritmos de análisis y síntesis}

Los siguientes algoritmos realizan las operaciones de análisis y síntesis en el dominio de las frecuencias y están basados en el empleo de la wavelet expuesta y en la FFT.

\section{Algoritmo 1}

- Paso 1: Se utiliza FFT para calcular la transformada de Fourier de la señal discreta $f(n), n=1, \cdots, 2^{N}$ obteniendo $\widehat{f}(m), m=1, \cdots, 2^{N}$.

- Paso 2: Se elige $\alpha=\pi / m, m \geq 3$ para la construcción de la ventana y la obtención de la transformada de Fourier de la wavelet madre, fórmula (1).

- Paso 3: Se elige nivel $j<0$ y se hallan los correspondientes coeficientes wavelet utilizando $\widehat{f}(k) \cdot \widehat{\psi}^{*}(k)$. 
- Paso 4: Se obtiene la correspondiente componente transformada $\widehat{f}_{j}(m)=$ $\sum_{k} c_{j k} \widehat{\psi}_{j k}(m)$.

- Paso 5: Se utiliza FFT para obtener $f_{j}$.

- Paso 6: Se repiten pasos 3 a 5 para obtener la descomposición de la señal $f(n)$.

\section{Algoritmo 2}

- Paso 1: Se elige a en función de la cantidad apropiada de subwavelets a aplicar.

- Paso 2: Se elige nivel $j<0$ y se utiliza $\widehat{f}_{j}$.

- Paso 3: Se obtienen las subcomponentes $\widehat{f_{j}^{(p)}}$, fórmulas (4) y (5).

- Paso 4: Se utiliza FFT para obtener $\widehat{f_{j}^{(p)}}$.

- Paso 5: Se obtienen las componentes $f_{j}$, fórmula (5).

\section{Conclusiones}

En este trabajo presentamos una nueva familia de funciones elementales infinitamente oscilantes y de banda limitada en el contexto de un Análsis de Multirresolución y el correspondiente algoritmo de análisis y síntesis, permitiendo descomponer las proyecciones sobre los subespacios wavelet en ondas casimonocromáticas, mejorando la precisión en frecuencia.

En algunas aplicaciones se requiere de wavelets infinitamente oscilantes, ortogonales, bien localizadas en tiempo y frecuencia, tal es el caso de la implementación de algoritmos de wavelet leaders para análisis de regularidad y formalismo multifractal o para la resolución de ecuaciones integro-diferenciales [1], [3].

\section{Referencias}

[1] Donoho, D.L. (1995) "Nonlinear solution of Linear Inverse problems by Wavelet-Vaguelet decomposition", Applied and Computational Harmonic Analysis 2(2): 101-126. 
[2] Huang, N.E; Shen, Z.; Long, S.R.; Wu, M.C.; Shih, H.H.; Zheng, Q.; Yen, N.C.; Tung, C.C.; Liu, H.H. (1998) "The empirical mode decomposition and the Hilbert spectrum for non-stationary time series analysis", Proc. $R$. Soc. Lond. A 454: 903-995.

[3] Jaffard, S.; Lashermes, B.; Abry, P. (2007) "Wavelet leaders in multifractal analysis", in: T. Qian, M. Vai, X. Yuesheng (Esd.) Wavelet Analysis and Applications, Birkhäuser, Basel, Switzerland: 201-246.

[4] Li, L.C. (2010) "A new method of wavelet transform based on FFT for signal processing", Second WRI Global Congres on Intelligent Systems, IEEE Computer Society: 203-206.

[5] Mallat, S. (2009) A Wavelet Tour of Signal Processing, The Sparse Way. Academic Press-Elsevier, Burlington MA.

[6] Meyer, Y. (1993) Wavelets, Algorithms and Applications. SIAM, Philadelphia PA.

[7] Meyer, Y. (2001) Oscillating Pattern in Image Processing and Nonlinear Evolution Equations. American Mathematical Society, Providence RI.

[8] Serrano, E.; Figliola, A. (2008) Littlewood-Paley spline wavelets: a simple and efficient tool for signal and image processing in industrial applications, Proceedings in Applied Mathematics and Mechanics (PAMM), Wiley InterScience, 7: 1040313-1040314.

[9] Serrano, E.; Fabio, M. (2010) "Diseño de funciones elementales combinando la transformada wavelet y la transformada de Hilbert", UMA 2010, Tandil, Argentina.

[10] Serrano, E.; Fabio, M.; Aragón, A. (2011) "Caracterización de la frecuencia instantánea en señales tipo pasa-banda", III MACI, Asociación Argentina de Matemática Aplicada, Computacional e Industrial. Bahía Blanca, Argentina. 
